\title{
TEMPERATURE STRUCTURE IN MERGING COMA CLUSTER OF GALAXIES
}

\author{
H. HONDA
}

The Institute of Space and Astronautical Science, 3-1-1, Yoshinodai, Sagamihara, Kanagawa, 229, Japan

M. HIRAYAMA, H. EZAWA

Department of Physics, University of Tokyo, 7-3-1 Hongo, Bunkyo-ku, Tokyo 113, Japan

K. KIKUCHI, T. OHASHI

Department of Physics, Tokyo Metropolitan University, Hachioji, Tokyo 192-03, Japan

AND

M. WATANABE, H. KUNIEDA, K. YAMAHITA

Department of Physics, Nagoya University, Chikusa-ku, Nagoya 464-01, Japan

\section{Introduction}

The Coma cluster has been recognized as an archetype of rich and relaxed clusters, until recent ROSAT observations reveal that the intracluster medium (ICM) has a complex distribution (Briel et al. 1992; White et al. 1993). The X-ray surface brightness distribution shows a secondary peak around the galaxy NGC 4839 , at $40^{\prime} \mathrm{SW}$ from the cluster center.

Works by Hughes (Hughes et al. 1988a; Hughes et al. 1988b; Hughes 1989 ) show that the core of the cluster is nearly isothermal out to a radius of $1^{\circ}$ and suggest that the temperature falls with radius beyond that. Hughes (1993), based on the recent analysis of the Ginga scanning observations, shows that the ICM is approximately isothermal in the core and becomes cooler beyond several core radii. Here, we report on the recent mapping observations of the Coma cluster with ASCA (see Honda et al. 1996 for details). 


\section{Analysis and Results}

The observed data can be regarded as a weighted sum of the whole cluster emission, and one need to know the complete distribution of the surface brighness and temperature all over the cluster in order to derive the temperature in a given position. In principle, this is obtained by solving the whole temperature map in a self consistent way. Therefore, we are developing the new analysis system called TERRA (Kikuchi et al. in this volume).

Using the TERRA system, we obtain significant variation of the temperature. The temperature is lower than $4.5 \mathrm{keV}$ in the west region and higher than $10 \mathrm{keV}$ in the east one, respectively, both offset by $40^{\prime}$ from the center. Systematic drop of the temperature in the outer region of the cluster has been observed in other systems.

\section{Temperature structure}

The azimuthal variation of temperature is explained that while central regions of these clusters have been heated up due to recent mergers, the disturbance is not reaching the outer parts of the clusters. In other words, these cool outer gas may be under a pre-merger equilibrium state.

When a merger occurs, dark matter particles take a longer time than the gas to settle into a sigle body. This is seen in numerical simulations (see e.g. Evrard 1990). In a cluster experiencing a merger, diffence in spatial distribution between gas and dark matter would be larger than that in relaxed clusters. Since dark matter is the dominant source of gravitational potential, part of the gas may be pulled by the extended dark matter field. Such an effect may cause temperature variation in the outer part of the cluster. Detailed calculation or simulation would be necessary to look into these possibilities. Mapping observation with ASCA of other rich clusters would be useful to obtain more knowledge on mergers and cluster evolutions.

\section{References}

1. Briel, U. G., Henry, J. P., and Boehringer, H. 1992, A\&A, 259, L31

2. Evrard, A.E. 1990, Clusters of Galaxies, ed. W.R. Oegerle (Cambridge: Cambridge Univ. Press), 287

3. Honda, H., Hirayama, M., et al. 1996, ApJ, 473, L71

4. Hughes, J.P., Butcher, J. A., Stewart, G. C., and Tanaka, Y. 1993, ApJ, 404, 611

5. Hughes, J.P. 1989, ApJ, 337, 221

6. Hughes, J.P., Gorenstein, P., and Fabricant, D. 1988a, ApJ, 329, 82

7. Hughes, J.P., et al. $1988 \mathrm{~b}$, ApJ, 327, 615 